\title{
Access, Bypass and Productivity Gains in Competitive Postal Markets
}

Axel Gautier, HEC, Université de Liège and CORE, Université Catholique de Louvain, agautier@ulg.ac.be

Francis Bloch, GREQAM, Université de la Méditerranée and Warwick University, francis.bloch@univmed.fr

Published in Michael A. Crew and Paul R. Kleindorfer, (2009). Competition and Regulation in the Postal and Delivery Sector, Edward Elgar, Northampton, MA, USA and Cheltenham, UK.

\section{INTRODUCTION}

In the European Union, Full Market Opening (FMO) of postal markets is now scheduled for

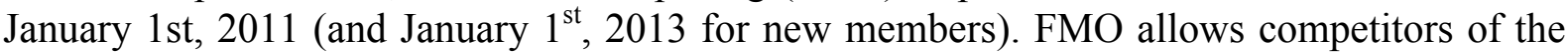
incumbent postal operator to enter all the segments of the postal markets including mail delivery. FMO might be a threat for the financing of the Universal Service Obligation (USO) imposed on the incumbent postal operator, particularly if the entrant bypasses the incumbent's delivery network.

In the postal sector, competition has started long before FMO. However, with a few exceptions, competition was limited to the upstream segments of the market (collection and sorting) through the use of worksharing agreements. Worksharing or access means that a competitor can perform all the upstream operations of the postal value chain and buy access to the incumbent's PO delivery network at a discounted price (compared to the letter price). FMO means that in addition to the access solution that will continue to be available, the competitors also have the option to deliver mail with their own delivery network (bypass).

In this paper, we are interested in the consequences of FMO on the behavior of the incumbent PO. As mentioned, FMO means that the entrant can bypass the incumbent's delivery network and build up its own. Bypass can be detrimental for both the incumbent's profit -with a negative impact on its ability to finance the USO- and welfare (Crew and Kleindorfer 2005, De Donder 2006, and Bloch and Gautier 2007). Our interest lies in the strategies that the incumbent can develop to prevent (inefficient) bypass by the entrant. In particular, we concentrate on the pricing and on the investment strategies undertaken by the incumbent (or the regulator) to deter bypass.

In this paper, we will consider how the incumbent's strategies will be modified by the threat of bypass by the entrant. To this end, we construct a model where an incumbent PO competes in postal markets with a profit-maximizing entrant. The entrant chooses its letter price and the delivery method -access or bypass- that leads to the highest profit. As a starting point of our analysis, we consider the prices and investment level that apply when competition is limited to the upstream segments of the market. These pre-FMO prices are either set by a welfaremaximizing regulator or by a profit-maximizing firm. We show that, facing the threat of entry, the incumbent PO may change its price structure and may also strategically invest in its delivery network,

By changing its prices, the incumbent can strategically deter bypass by making this option less profitable for the entrant. To do so, the incumbent manipulates both its letter and access 
prices. ${ }^{1}$ The two instruments affect differently the incentives to bypass. For the entrant, the access price is the cost of outsourcing delivery. Therefore a change in the access price amounts to a change in production cost. To deter bypass, the incumbent can thus lower its access price to make the access option relatively more profitable for the entrant.

A change in the letter price changes the volume of mails carried by the entrant. The lower the incumbent's letter price, the lower the demand faced by the entrant. And the magnitude of this effect depends on the cross-price elasticity of the demands. But this holds true under both access and bypass. So, how can the incumbent deter bypass by changing its letter price? Suppose that a given price change has the same impact on the entrant's mailing volume under access and bypass. Then, the impact of a price change on its profit depends on the price cost margin in the two cases. If the unit margin is highest under bypass, a price cut by the incumbent leads to a higher profit decrease with bypass. Therefore, decreasing the letter price makes the access option more attractive. Conversely, if the unit margin is highest under access, the incumbent will deter bypass by increasing its letter price i.e. by reducing the entrant's volume. In this paper, we show that the incumbent PO must reduce both the access and the delivery prices to prevent bypass. However, constraining the entrant to choose access is costly in terms of foregone profit and welfare since the incumbent departs from pre-FMO prices, which are assumed to be efficient.

Next, we introduce the possibility for the incumbent PO to increase the productivity of its delivery activity by restructuring it. For example it could re-organize its delivery network or invest to expand automated sorting. The incentives to undertake these kinds of investment depend on the expected gains that are obviously affected by the delivery method chosen by the entrant. We can decompose the benefit of the investment into a direct and a strategic component. The size of the investment depends on the magnitude of these two components. The direct effect is linked to the mail volume processed by the incumbent. With a larger mail volume, the gains from reducing by a given amount the unit delivery cost are larger. Therefore the direct effect is linked to number of letters delivered by the incumbent, including those pre-sorted by the entrant and posted at an access point. Regarding the direct incentive only, the incumbent invests more under access than under bypass since the latter implies a (possibly drastic) reduction in the number of postal items delivered. The strategic effect is linked to the impact of the investment on the competitor's behavior (see Fundenberg and Tirole, 1984 for a related analysis). In this context, the only change in behavior that the incumbent can expect from the entrant is a change of delivery method. Because of these strategic incentives, the incumbent PO may invest more to deter bypass. Hence, the investment together with its pricing decisions will be used by the incumbent to deter bypass by the entrant.

We show that it is not when the two firms are engaged in so-called facility based competition that the incentive to invest are the highest. The incumbent firm has the highest incentive to invest when its investment can modify the delivery decision of the entrant. Hence, we will observe the highest investment levels for the firms that try to deter their competitor from engaging in facility-based competition.

\footnotetext{
${ }^{1}$ In Bloch and Gautier (2007), the entrant is competitive and chooses the cheapest option for mail delivery. Therefore the letter price does not influence the delivery decision and the only possible way to deter bypass is to make access less costly than bypass i.e. to reduce the access price.
} 
The paper is organized as follow: we present the basic ingredients of the model in section 2 . We derive the post-FMO price changes in section 3. Section 4 considers the investment incentives and section 5 concludes.

\section{MODEL}

The incumbent PO, denoted hereafter firm 1, offers two products: an E2E letter at price $p_{1}$ and access to its delivery network at price $a$. The entrant, firm 2, offers only an E2E letter at price $p_{2}$. Consumers view the two E2E letter products as imperfect substitutes. For example, the entrant may have a lower frequency of collection or delivery and/or a lower geographical coverage.

The demand for the E2E letter offered by firm $i=1,2$ is denoted $x_{i}\left(p_{1}, p_{2}\right)$. These demand functions have the standard properties. The entrant has two options for mail delivery. It can either buy access at unit-price $a$ or it can set-up its own delivery network. In case it chooses the access option, the demand for access is $x_{2}\left(p_{1}, p_{2}\right)$. In case it chooses to bypass, there is no demand for access and the incumbent sells only an E2E letter to consumers. This means that consumer direct access is not considered in this model.

We decompose the postal value chain in two composite activities: upstream activities (collection, transport, sorting) and delivery (distribution). The constant unit cost of all the upstream activities is denoted by $c_{i}$ for firm $i$. Delivery involves two types of cost: a fixed cost and a constant per-unit cost. The fixed costs are denoted by $F_{1}$ and $F_{2}$, the per-unit cost $d_{1}$ and $d_{2}$. The entrant can avoid these delivery costs, if it chooses to buy access from the incumbent. In addition, universal service obligations imposed on the incumbent operator involves a fixed $\operatorname{cost} F$.

Firms compete in prices. We suppose that the incumbent is price leader. This means that the entrant decides on its price $p_{2}$ and on its delivery method after observing the letter and the access prices set by the incumbent. The entrant chooses the price and the delivery option that maximize its profits. ${ }^{2}$ The prices of the incumbent are either set by the firm in order to maximize its profits or by a postal regulator that seeks to maximize welfare.

In the regulated case, the regulator can decentralize the welfare maximizing prices by imposing a global price cap on the incumbent (Billette de Villemeur et al., 2003). The main difference between regulated and un-regulated prices is the price levels and not the price structure. From now on, we adopt the convention that the incumbent and not the regulator sets the prices in order to maximize its profit (in the absence of a price cap) or to maximize welfare.

\section{PRICING}

\subsection{Pre-FMO pricing}

\footnotetext{
${ }^{2}$ Other models of the mail sector typically assume that the entrant behaves as a competitive fringe and sets its price at marginal cost. This assumption of competitive fringe seems extreme. We consider here another extreme assumption - von Stackelberg leadership- where the entrant chooses its follower price in order to maximize profits. The reality of competition between the PO and the entrant lies probably somewhere between these two extreme assumptions.
} 
Consider the pre-FMO situation where the entrant does not have the possibility to bypass the incumbent's delivery network. The game played by the incumbent and the entrant is a twostage game. At a first stage, the incumbent sets its retail (stamp) price $\hat{p}_{1}$ and its access price $\hat{a}$. At a second stage, after observing these prices, the entrant sets its retail (stamp) price $\hat{p}_{2}\left(\hat{p}_{1}, \hat{a}\right)$.

In this paper, we leave aside the exact description of the optimal prices. This work has been done elsewhere by Laffont and Tirole (1994) or Crew and Kleindorfer (1992), Bloch and Gautier (2007) and Billette de Villemeur et al. (2007) for the postal sector. For our argument, it is sufficient to consider the maximization problems faced by the two firms. ${ }^{3}$ The entrant chooses its price $\hat{p}_{2}^{A}\left(\hat{p}_{1}, \hat{a}\right)=\underset{p_{2}}{\arg \max _{2}} \Pi_{2}^{A} \equiv\left(p_{2}-c_{2}-\hat{a}\right) x_{2}\left(\hat{p}_{1}, p_{2}\right)$.

We let $Z^{A}$ denote the objective of the incumbent, where $Z^{A}$ equals profit in the absence of a price-cap and welfare otherwise. The optimal prices $\hat{p}_{1}$ and $\hat{a}$ are the solutions of:

$$
\begin{aligned}
& \frac{\partial Z^{A}}{\partial p_{1}}+\frac{\partial Z^{A}}{\partial \hat{p}_{2}} \frac{\partial \hat{p}_{2}}{\partial p_{1}}=0 \\
& \frac{\partial Z^{A}}{\partial a}+\frac{\partial Z^{A}}{\partial \hat{p}_{2}} \frac{\partial \hat{p}_{2}}{\partial a}=0
\end{aligned}
$$

In particular, we note that the incumbent chooses its stamp and access prices taking into account their effects on the optimal choice of the entrant.

\subsection{Post FMO pricing}

FMO means that the entrant has the possibility of bypassing the incumbent's delivery network. Under FMO, the game played by the incumbent and the entrant becomes a threestage game. First, as before, the incumbent sets its prices. Second, the entrant decides on its delivery method -access or bypass-. And, third, the entrant sets its retail price.

Consider the last stage of this game. In case of access, the entrant chooses the price $\hat{p}_{2}^{A}$. In case of bypass, its optimal price is found by solving $\hat{p}_{2}^{B}\left(\hat{p}_{1}, d_{2}\right)=\arg \max _{p_{2}} \Pi_{2}^{B} \equiv\left(p_{2}-c_{2}-d_{2}\right) x_{2}\left(\hat{p}_{1}, p_{2}\right)-F_{2}$

The optimal delivery choice of the entrant at the second stage of the game is easily found. If $\Pi_{2}^{B}>\Pi_{2}^{A}$, the entrant will choose to bypass. If $\Pi_{2}^{B}<\Pi_{2}^{A}$, it will choose access, and if $\Pi_{2}^{B}=\Pi_{2}^{A}$, it will be indifferent between the two delivery methods. Obviously, the parameters of the delivery function of the entrant only affect profit under bypass. Hence, comparing the profit levels in the two cases, we can easily establish the following:

Proposition 1: At any prices $\left(p_{1}, a\right)$, there exists a cut-off level for the entrant's marginal delivery cost $\widetilde{d}_{2}$ such that (1) the entrant prefers bypass for $d_{2}<\widetilde{d}_{2}$ and access otherwise, (2) $\widetilde{d}_{2}<a$ and (3) the value of $\widetilde{d}_{2}$ decreases with the fixed cost $F_{2}$.

\footnotetext{
${ }^{3}$ We will assume that the second order conditions hold for all maximization problems, namely that the entrant's profit, $\Pi_{2}$, is concave in $p_{2}$ and the incumbent's objective, $Z^{\mathrm{A}}$ is concave in $a$ and $p_{1}$.
} 
PROOF: At given prices $\left(p_{1}, a\right)$, the profit $\Pi_{2}^{B}$ decreases in $d_{2}$. The profit $\Pi_{2}^{A}$ is independent of $d_{2}$. Therefore, there exist a level $\widetilde{d}_{2}$ such that $\Pi_{2}^{B}=\Pi_{2}^{A}$. For $d_{2}=a, \Pi_{2}^{B}-\Pi_{2}^{A}=-F_{2}$. Hence, $\widetilde{d}_{2}$ is clearly smaller that the access price level.

In a liberalized market, the entrant has two available technologies for mail delivery: a constant return to scale one -access- available at price $\hat{a}$ and an increasing return to scale one -bypasscharacterized by a fixed cost $F_{2}$ and a constant unit cost $d_{2}{ }^{4}$. Because of the fixed cost, the entrant does not choose the technology with the lowest marginal cost. That is for $d_{2} \in\left[\widetilde{d}_{2}, \hat{a}\right]$, the marginal delivery cost is lower under bypass but, because of the fixed cost, the highest profit is achieved if the entrant buys access.

Proposition 1 clarifies the conditions on the parameters of the model under which access or bypass is likely to occur. We now return to the first stage of the model, keeping the delivery costs $d_{2}$ and $F_{2}$ fixed, and computing the optimal choice of the incumbent.

\subsection{Access, bypass and constrained access}

Formally, our model is similar to the classical model of entry barriers, where the incumbent chooses a price in order to block, deter or accommodate entry of a competitor (Tirole (1988), Chapter 8). The model differs from the classical model in two respects: (i) the choice of the entrant is not whether to enter or not, but whether to bypass or use the delivery technology of the incumbent, and (ii) accordingly, the incumbent chooses two instruments rather than one, namely both an access price $a$ and a delivery price $p_{l}$.

By analogy with the model of entry barriers, we can distinguish three regimes:

Access (blocked bypass): By setting its optimal delivery and access prices, the incumbent prevents bypass.

Constrained access (deterred bypass): The incumbent distorts its delivery and access prices in order to force the entrant to choose access.

Bypass (accommodated bypass): The incumbent prefers to let the entrant set up its own delivery network, and chooses its optimal prices anticipating bypass.

As in the model of entry barriers, some remarks are in order. First, the choice of the incumbent depends crucially on the 'entry' $\left(F_{2}\right)$ and delivery $\left(d_{2}\right)$ costs of the entrant. The higher these costs, the less likely the entrant is to choose bypass. Hence, when entry and delivery costs are high enough, bypass is always blocked. When they are very low, the incumbent prefers to accommodate bypass, and chooses its optimal prices accordingly. Constrained access appears only for intermediate values of the entry and delivery costs. Second, the analysis is conducted under the assumption that the incumbent can commit to its access and delivery prices. Absent this commitment, the incumbent would be unable to influence the entrant's decision, and the analysis would be markedly different: the incumbent and entrant would both choose Bertrand equilibrium prices.

\footnotetext{
4 In Bloch and Gautier (2007), we assumed $F_{2}$ to be zero. In this case, the entrant can choose between two constant returns to scale technologies for mail delivery. Clearly, it chooses the cheapest one: access for $d_{2} \geq a$ and bypass otherwise.
} 


\subsection{Constrained access}

We now focus attention on the regime of strategic bypass deterrence, when the incumbent chooses its delivery and access prices in order to force the entrant to choose access. To do that, the incumbent must ensure that the following constraint is satisfied.

$$
\left(\hat{p}_{2}^{B}\left(p_{1}, d_{2}\right)-c_{2}-d_{2}\right) x_{2}\left(p_{1}, \hat{p}_{2}^{B}\left(p_{1}, d_{2}\right)\right)-F_{2} \leq\left(\hat{p}_{2}^{A}\left(p_{1}, a\right)-c_{2}-a\right) x_{2}\left(p_{1}, \hat{p}_{2}^{A}\left(p_{1}, a\right)\right)
$$

This equation shows that the incumbent can use either of his two instruments (access or delivery price) to prevent bypass. Clearly, at the optimal delivery price, the incumbent needs to reduce its access price (with respect to the optimal access price) in order to deter bypass. Similarly, at the optimal access price (which satisfies $\hat{a}>d_{2}$, since the entrant prefers bypass at the optimal pre-FMO prices), the incumbent needs to reduce the delivery price, in order to lower mail volume thereby reducing the mail volume of the entrant, and the relative advantage of bypass over access.

The previous heuristic argument shows that, if the incumbent sets either instrument at the optimal pre-FMO level, it will necessarily choose to lower the other price with respect to the optimal pre-FMO level. Hence, one expects that, in the constrained access regime, the full opening of the postal markets will induce a decrease in the delivery price of the PO or the access price charged to the entrant. What if the incumbent can now independently vary the two prices?

Formally, the optimal delivery and access prices in the constrained access regime will be chosen by the incumbent in order to maximize its objective function $\mathrm{Z}^{\mathrm{A}}$ subject to the constrained access constraint (1). If we denote by $\mu$ the Lagrange multiplier of this constraint, the first-order conditions of the profit/welfare maximization problem read as follows:

$$
\begin{aligned}
& \frac{\partial Z^{A}}{\partial p_{1}}+\frac{\partial Z^{A}}{\partial \hat{p}_{2}} \frac{\partial \hat{p}_{2}}{\partial p_{1}}+\mu\left(\left(\hat{p}_{2}^{A}-c_{2}-a\right) \frac{\partial x_{2}\left(p_{1}, \hat{p}_{2}^{A}\right)}{\partial \hat{p}_{2}} \frac{\partial \hat{p}_{2}^{A}}{\partial p_{1}}-\left(\hat{p}_{2}^{B}-c_{2}-d_{2}\right) \frac{\partial x_{2}\left(p_{1}, \hat{p}_{2}^{B}\right)}{\partial \hat{p}_{2}} \frac{\partial \hat{p}_{2}^{B}}{\partial p_{1}}\right)=0 \\
& \left.\frac{\partial Z^{A}}{\partial a}+\frac{\partial Z^{A}}{\partial \hat{p}_{2}} \frac{\partial \hat{p}_{2}}{\partial a}+\mu\left(-x_{2}\left(p_{1}, \hat{p}_{2}^{A}\right)+\left(\hat{p}_{2}^{A}-c_{2}-a\right) \frac{\partial x_{2}\left(p_{1}, \hat{p}_{2}^{A}\right)}{\partial \hat{p}_{2}} \frac{\partial \hat{p}_{2}}{\partial a}\right)\right)=0
\end{aligned}
$$

Let $\bar{p}_{1}$ and $\bar{a}$ be the solutions to this problem. We can establish the following:

Proposition 2: To deter bypass, (1) the access price must be lowered $\bar{a} \leq \hat{a}$ and (2) the incumbent's letter price must be decreased $\bar{p}_{1} \leq \hat{p}_{1}$.

PROOF: If at prices $\left(\hat{p}_{1}, \hat{a}\right)$, the constraint (1) is slack, $\mu=0$ and bypass can be prevented with the pre-FMO prices (blocked bypass case). Otherwise if the prices $\left(\hat{p}_{1}, \hat{a}\right)$ induce bypass, prices must be changed and (1) is binding $(\mu>0)$.

(1) The term $\left(-x_{2}\left(p_{1}, \hat{p}_{2}^{A}\right)+\left(\hat{p}_{2}^{A}-c_{2}-a\right) \frac{\partial x_{2}\left(p_{1}, \hat{p}_{2}^{A}\right)}{\partial \hat{p}_{2}} \frac{\partial \hat{p}_{2}}{\partial a}\right)$ is negative. Hence, when the constraint is binding $\mu>0$, the access price is below its optimal level $\hat{a}$.

(2) If $\mu>0$, we have $a>d_{2}$ (see proposition 1). This implies that the term $\left(\left(\hat{p}_{2}^{A}-c_{2}-a\right) \frac{\partial x_{2}\left(p_{1}, \hat{p}_{2}^{A}\right)}{\partial \hat{p}_{2}} \frac{\partial \hat{p}_{2}^{A}}{\partial p_{1}}-\left(\hat{p}_{2}^{B}-c_{2}-d_{2}\right) \frac{\partial x_{2}\left(p_{1}, \hat{p}_{2}^{B}\right)}{\partial \hat{p}_{2}} \frac{\partial \hat{p}_{2}^{B}}{\partial p_{1}}\right) \quad$ is negative. Hence, when $\mu>0$, the price $\bar{p}_{1}$ must be lower than $\hat{p}_{1}$. 
Hence, in general, in order to prevent bypass, both the delivery and access prices will be adjusted downwards. The access price is reduced to increase the margin of the entrant and make access more attractive. The delivery price is lowered in order to reduce the mail volume carried by the entrant, again making bypass less attractive.

We now analyze how changes in the delivery and entry cost of the entrant affect the optimal value of the incumbent's objective.

Proposition 3: In the constrained access regime, when the costs of the entrant, $F_{2}$ and $d_{2}$, increase, the optimal value of the incumbent's objective increases. Furthermore, either the delivery or the access price of the incumbent must go up.

PROOF: The second part of the Proposition is easily proved, by looking at constraint (1). If $F_{2}$ increases and $a$ and $p_{1}$ both decrease, the difference between access and bypass must strictly increase - contradicting the equality of constraint (1). Similarly, when the delivery cost $d_{2}$ increases, one of the two optimal prices of the incumbent prices must go up. This holds true as long as (1) is binding. Prices will increase with the cost of the entrant up to their pre-FMO level. Once they reach that level, the constraint (1) is slack and we switch from the constrained access case to the access (blocked bypass case).

For the first part of the proposition, notice that an increase in the entry or delivery costs relaxes constraint (1): any pair of prices $\left(\bar{p}_{1}, \bar{a}\right)$ which was feasible under the old parameter values will remain feasible under the new values. Hence, the objective of the incumbent can never decrease, and will in fact generally increase, as the set of prices for which bypass is dominated by access will be strictly enlarged when $F_{2}$ and $d_{2}$ increase.

We thus conclude that the objective of the incumbent under the constrained access regime is a strictly increasing function of the delivery and entry costs of the entrant. When these values are high enough to guarantee that the entrant prefers to access the incumbent's network, blocked bypass is obtained. Furthermore, as in the classical model of barriers to entry, bypass deterrence will become more and more costly as the entry and delivery costs of the entrant go down. For low values of $F_{2}$ and $d_{2}$, the PO will prefer to accommodate bypass and let the entrant build its own delivery network.

\section{INVESTMENT}

In this section, we introduce the possibility for the incumbent firm to invest in a cost-reducing delivery technology before it fixes its prices. Consider that, before fixing its prices, the incumbent can undertake a costly investment that reduces its unit delivery cost $d_{l}$. Precisely, we consider that by investing an amount $\phi(e)$, the incumbent PO reduces its marginal delivery cost by an amount $e$. The function $\phi(e)$ is increasing and convex in $e$ and the incumbent decides on the investment size $e$.

Increasing the productivity of the delivery network is a key challenge for postal operators in the perspective of FMO. ${ }^{5}$ The recent PWC report (2006) recognizes that there is a significant

\footnotetext{
5 Increasing the productivity of the incumbent PO is a way to finance (at least partially) the USO in a competitive postal market. Cremer et al. (2002) is an early model showing the relationship between productivity improvements and for the ability of the incumbent to meet its universal service obligations after FMO.
} 
potential for productivity growth in European PO and list some initiatives that can be put in place in order to increase efficiency (page 62). Among those, the followings recover the above idea of investments that reduce the variable cost of delivery: sorting automation and/or rationalization of transport and sorting networks, centralized sorting centers, walk sequencing and/or walk route optimization. The size of these investments can be considerable; For example, the Austrian Post invested 200 million euros in the reduction (from 39 to 6) and the automation of its sorting centers.

If the competitor chooses access, the profit of the incumbent is equal to $\Pi_{1}^{A}=\left(p_{1}-c_{1}-d_{1}\right) x_{1}\left(p_{1}, \hat{p}_{2}^{A}\right)+\left(a-d_{1}\right) x_{2}\left(p_{1}, \hat{p}_{2}^{A}\right)-F_{1}-F$ and if the competitor chooses to bypass profit is $\Pi_{1}^{B}=\left(p_{1}-c_{1}-d_{1}\right) x_{1}\left(p_{1}, \hat{p}_{2}^{B}\right)-F_{1}-F$. Let $\hat{\Pi}_{1}^{K}$ be the value of the profit of firm 1 under access $(\mathrm{k}=\mathrm{A})$ and bypass $(\mathrm{k}=\mathrm{B})$ evaluated at the optimal prices. In the absence of a price cap, the investment level is determined by maximizing $\hat{\Pi}_{1}^{K}-\phi(e)$ with respect to $e$. In the accommodated and blocked bypass cases, the optimal value of $e$ is found by solving: $\partial\left(\hat{\Pi}_{1}^{K}-\phi(e)\right) / \partial e=0$. Taking the total derivative with respect to $e$ and using the envelope theorem, the optimal investment level in the access and bypass cases are:

$$
\begin{array}{cl}
e^{A}=\phi^{-1}\left(x_{1}+x_{2}\right) & \text { If the entrant chooses access } \\
e^{B}=\phi^{-1}\left(x_{1}\right) & \text { If the entrant bypasses }
\end{array}
$$

This means that in the situations in which the incumbent accommodates bypass or access, the optimal investment size is directly linked to the volume of mail processed, including the mail of the competitors that are pre-sorted and posted at an access point. So mail volume is a key determinant of investment. The larger the volume, the higher the profit increase for a given reduction in the per-unit delivery cost. We will refer to direct incentives as the investment incentives linked to the mail volume.

Given the observed price elasticities and displacement ratios in the postal sector, it is likely that the investment level would be higher when the entrant chooses the access option than when it chooses to bypass since in the latter case, the incumbent looses the volume $\mathrm{x}_{2}$ which reduces the incentives for investment.

Next, consider the situations where after FMO, the incumbent blocks bypass. In the constrained access region, the incumbent will manipulate its delivery and access prices to prevent bypass. This distortion will clearly affect the incentives to invest in a technology to reduce delivery cost. In fact, we now have the following. The derivative of $\Pi_{1}^{A}-\phi(e)$, evaluated at the optimal prices $\left(\bar{p}_{1}, \bar{a}\right)$, with respect to $e$ writes as follows:

$$
\phi^{\prime}(e)=x_{1}+x_{2}+\left(\frac{\partial \Pi_{1}^{A}}{\partial p_{1}}+\frac{\partial \Pi_{1}^{A}}{\partial p_{2}} \frac{\partial p_{2}}{\partial p_{1}}\right) \frac{\partial p_{1}}{\partial d_{1}}+\left(\frac{\partial \Pi_{1}^{A}}{\partial a}+\frac{\partial \Pi_{1}^{A}}{\partial p_{2}} \frac{\partial p_{2}}{\partial a}\right) \frac{\partial a}{\partial d_{1}}
$$

Given the constraint (1) faced by the incumbent, the derivatives $\left(\frac{\partial \Pi_{1}^{A}}{\partial p_{1}}+\frac{\partial \Pi_{1}^{A}}{\partial p_{2}} \frac{\partial p_{2}}{\partial p_{1}}\right)$ and $\left(\frac{\partial \Pi_{1}^{A}}{\partial a}+\frac{\partial \Pi_{1}^{A}}{\partial p_{2}} \frac{\partial p_{2}}{\partial a}\right)$ are no longer equal to zero. In fact, applying the same reasoning as above, we can easily show that these derivatives are strictly positive (see proposition 2 ). Hence, there are two additional terms which increase the level of investment in the constrained access case. We will refer to these additional terms as the strategic incentives for investment. 
In the constrained access case, there is still a direct incentive for investment which is linked to the mail volume. But, there is in addition a strategic incentive. It captures the impact of the investment on the induced behavior of the entrant. The strategic incentives capture the impact of decreasing $d_{l}$ on the distortions in prices that the incumbent applies to deter bypass.

To understand this, notice that in order to prevent bypass, the incumbent reduces its access price $a$. If the incumbent reduces its delivery cost $d_{1}$, then the optimal pre-FMO access price also decreases. Hence, investing in the delivery network reduces the optimal access price and thus constitutes an alternative way to prevent bypass. ${ }^{6}$ The incumbent also changes its letter price in the constrained access region. We know that a lower delivery cost implies a lower delivery price $p_{l}$. Hence, as it is optimal to reduce delivery price to deter bypass, investing in a delivery network makes the letter price reduction less costly. It therefore fosters the investment incentives.

On the other hand, if the incumbent chooses to accommodate bypass, incentives to invest decline sharply, because the incumbent now only faces a direct effect on its own mail volume, $x_{1}$. Hence, we expect to observe an abrupt, discontinuous, decrease in the level of investment when the entry and delivery costs of the entrant are so low as to prevent the incumbent from deterring bypass. But this also shows that the incumbent can use investments in productivity gains in order to affect the delivery regimes. When delivery costs $d_{l}$ go down, the incumbent's incentives to maintain access and prevent bypass increase, so that the incumbent may have an extra incentive to invest, in order to switch from a regime of accommodated bypass to a regime of constrained access.

If a price cap is imposed on the incumbent $\mathrm{PO}$, the investment will depend on the same components -strategic and direct- incentives. However, the investment level will not be the same since the mail volume is changed when a price cap is imposed. In general, a quantitative analysis of the investment levels for a welfare-maximizing (or price-cap constrained) firm requires a detailed analysis of the demand functions. This is beyond the scope of this paper.

Up to now, we only considered investments that reduce the marginal cost of delivery. Investments that aim at reducing the fixed cost of delivery and/or the financial burden of the USO would also prove useful to deter bypass. Decreasing the scope of the USO and the associated cost would do the same. ${ }^{7}$ If the incumbent reduces its fixed costs, it can charge a lower mark-up over its costs to break-even. Hence, these types of investment contribute to reduce the price $p_{1}$ and $a$, and therefore they are also useful to prevent inefficient bypass.

\section{CONCLUDING REMARKS}

In this paper, we have shown that the incumbent PO may strategically deter bypass by modifying its price structure. By changing the access price, it manages to influence the cost of the entrant and by changing its letter price, it manages to modify the mail volume carried by the entrant. And, both the mail volume and the cost determine the decision to bypass or not. We also consider the possibility for the incumbent of engaging in a costly restructuring of its delivery activity. We identify the incentives to undertake such an investment and we show

\footnotetext{
${ }^{6}$ Preventing bypass by changing the price requires that the incumbent $\mathrm{PO}$ is able to commit on its price. There is no problem of this kind for the investment.

${ }^{7}$ Crew and Kleindorfer (2006) show that reducing the scope of the USO (reducing counter density) is optimal under FMO for a regulator that wants to avoid subsidies.
} 
that they are directly related to the mail volume distributed by the incumbent. Hence, it is when the entrant remains a monopolist in the delivery activity that the direct incentives are the strongest.

There is also a strategic motive for investment. In regions of the parameters for which the incumbent optimally deters bypass, a reduction of the delivery cost reduces the price distortion between the optimal price of the incumbent and its actual price. Hence, investing ex ante in a cost reducing investment makes it cheaper for the incumbent to deter bypass. This suggests that the incentives to invest in cost-reducing technologies may not be highest when the incumbent and the entrant are engaged in facility-based competition (accommodated bypass), but when the incumbent strategically distorts its delivery and access prices in order to deter bypass (deterred bypass). Hence, PO may be compelled to invest massively in costreducing technologies before FMO, in order to induce their competitors to choose access over bypass. In order to better understand the strategic effects of cost-reducing investments, it would be necessary to study in detail the actual patterns of investment by postal operators in Europe in anticipation of the full opening of the postal market.

\section{References}

Billette de Villemeur, Etienne, Cremer, Helmut Roy, Bernard and Joëlle Toledano, (2003), 'Optimal Pricing and Price-cap Regulation in the Postal Sector', Journal of Regulatory economics, 24 (1), 49-62.

Billette de Villemeur, Etienne, Cremer, Helmut, Roy, Bernard and Joëlle Toledano, (2007), 'Worksharing, Access and Bypass: the Structure of Prices in the Postal Sector', Journal of Regulatory Economics, 32(1), 67-85.

Bloch, Francis and Axel Gautier, (2007), 'Access Pricing and Entry in the Postal Sector', Review of Network Economics, forthcoming.

Cremer, Helmut, De Donder, Philippe and Frank Rodriguez, (2002), 'Funding the USO under Liberalisation: An Analysis of the Postal Market', in Postal and Delivery Services: Pricing, Productivity, Regulation and Strategy, edited by Crew, Michael A. and Paul R. Kleindorfer, Boston, MA: Kluwer Academic Publishers.

Crew, Michael A. and Paul R. Kleindorfer, (1992), The Economics of Postal Services, Boston, MA: Kluwer Academic Publishers.

Crew, Michael A. and Paul R. Kleindorfer, (2005), 'Competition, Universal Service and the Graveyard Spiral', in Regulatory and Economics Changes in the Postal and Delivery Sector, edited by Crew, Michael A. and Paul R. Kleindorfer, Boston, MA: Kluwer Academic Publishers.

Crew, Michael A. and Paul R. Kleindorfer, (2006), 'Approaches to USO under Entry', in Liberalization of the Postal and Delivery Sector, edited by Crew, Michael A. and Paul R. Kleindorfer, Cheltenham, UK and Northampton MA, USA: Edward Elgar.

De Donder, Philippe, (2006), 'Access Pricing in the Postal Sector: Theory and Simulations', Review of Industrial Organization, 28(3), 307-326. 
Fundenberg, Drew and Jean Tirole, (1984), 'The Fat-Cat Effect, the Puppy-Dog Ploy and the Lean and Hungry Look', American Economic Review, 74(2), 361-366.

Laffont, Jean-Jacques and Jean Tirole, (1994), 'Access Pricing and Competition', European Economic Review, 38(9), 1673-1710.

Price Waterhouse Coopers, (2006), 'The Impact on Universal Service of the Full Market Accomplishment of the Postal Internal Market in 2009', Report to the European Commission.

Tirole, Jean, (1988), The Theory of Industrial Organization, Cambridge and London: MIT Press. 